\title{
Differential Neural Network Online for Identification of an Electrocardiographic Signal
}

\author{
Héctor García Estrada ${ }^{1}$, Karen Jazmín Mendoza Bautista ${ }^{1}$, \\ Angelo Pastrana Manzanero ${ }^{1}$, Omar Alejandro Linares Escobar ${ }^{1}$, \\ María Guadalupe Ramírez Sotelo ${ }^{2}$, Agustín Ignacio Cabrera Llanos ${ }^{1}$ \\ ${ }^{1}$ Instituto Politécnico Nacional, Unidad Profesional Interdisciplinaria de Biotecnología, \\ Departamento de Bioprocesos, Mexico City, Mexico \\ ${ }^{2}$ Instituto Politécnico Nacional, Unidad Profesional Interdisciplinaria de Biotecnología, \\ Departamento de Bioingeniería, Mexico City, Mexico \\ aicllbuda@yahoo.com
}

\begin{abstract}
This work shows the development of a Differential Neural Network (DNN) applied to an online processing to signal from a 12-lead electrocardiograph. This system was divided in three stages: Acquisition of the ECG signal, design and tracking of the DNN. Firstly, a 12-lead electrocardiograph with a hybrid processing was constructed. Acquiring 8 leads with the USB DAQ-6009 and calculating the other four by programming. Then, based on the theory of Differential Neural Networks, the algorithm is developed, determining the values of the network by trial and error tests. To finally visualize the tracking of the network through our virtual instrument. The use of this technique is very common in the analysis of complex nonlinear dynamical systems in engineering and medicine areas, showed an excellent performance in the description of electrocardiographic biopotentials developed in this work.
\end{abstract}

Keywords: dynamic neural networks (DNN), electrocardiogram, LabVIEW, USB DAQ-6009, tracking of a biopotential.

\section{Introduction}

On the basis of Static Neural Networks (SNN) capability to approximate any nonlinear continuous function, a natural extension is to approximate the input-output behavior of nonlinear systems by Differential Neural Networks (DNN): their information process is described by diff erential equations for continuous time or by diff erence equations for discrete time. The existing results about this extension require quite restrictive conditions such as: an open loop stability or a time belonging to a close set.

This type of networks was developed based on the Lyapunov stability approach for the development of the laws of learning [1], these have had successful applications in fields such as biotechnology when estimating variables in a fermentation process [2], using observation schemes and using a technique that has been called virtual sensor [3]; in the estimation of drug doses for cancer, by generating a control signal in the dosage in such a way that the growth of cancer cells is not large [4].

Tracking is a phase of great importance and interest for the analysis and control of the process or systems, since we can, among other things, identify the parameters of the 
structure of the system to be studied and thus be able to design a model that behaves in a very similar to the system in question.

\subsection{Electrocardiograph}

An electrocardiograph is an electrical device that captures the electrical potentials of the heart by recording the voltage it generates and transmits through the body through a system of electrodes, cables and a recording console. These electrodes placed in specific parts of the body, are responsible for detecting cardiac depolarizations.

The heart cannot be seen from a single place, since it is a three-dimensional organ, so it must be from different places to be able to assess the electrical activity. Depending on the place where the explorer or derivative electrode is placed, they will be the electrical characteristics that we will appreciate.

The standard electrocardiogram consists of 12 leads, which can be divided into:

$$
\begin{aligned}
& \text { - Monopolar (aVR, aVL, aVF, V1 to V6) } \\
& \text { - Bipolar (DI, DII and DIII) }
\end{aligned}
$$

The monopolar derivations aVR, aVL and aVF, arose in 1942, when Goldberger observed that the signals of Wilson could be increased (since a $50 \%$ increase in the value of the detected signal is observed) if the average of the other points involved was taken. On the other hand, leads V1 to V6 complete the information needed to study the heart on almost all sides that provide information on how cardiac depolarization occurs according to what was previous mentioned.

The bipolar derivations, (also called standard) constitute a closed circuit (Kirchhoff's Law) and they comply with a law called Einthoven, which says that DII = DI + DIII, and is used to verify the correct placement of the cables [5].

\subsection{Differential Neural Networks (DNN)}

In the eighties J. Hopfield proposed the principle of operation of a stable recurrent network, which consists of a group of neurons where the output of each neuron serves as feedback at the entrances, except for its own entrance. This network arises from the theory of geometric control based on differential geometry [6].

The training process is an iterative process in which the input signals are applied, and the output is calculated; the process is repeated until the output signal is constant, which is when the network is said to be a stable network. In case that the output is not a constant output and is a variable output, you have an unstable network.

The continuous time Hopfield Neural Network or, on our terminology, the Diff erential Neural Networks, can be described by an electric circuit, which is based on a RC network connecting nonlinear amplifiers.

\subsection{Data Acquisition Device}

The device used to acquire data online is the NI USB DAQ-6009 card, which has a performance and sampling rate suitable for a certain number of applications that include 
control and automation of systems. Due to the use of this device, it was used to implement the software of graphic programming known as LabVIEW both company National Instrument. Same software has a group of exclusive components for this device, in which the user can acquire or generate various signals, which can be analog or digital [7].

\section{$2 \quad$ Metodology}

\subsection{Signal Acquisition}

For the acquisition of the signal it was necessary, firstly, the adequacy of the same, so it was designed as a 12-lead signal electrocardiograph. For this, an amplification was performed with the necessary gain to be acquired by the card, for which the AD620 instrumentation amplifier was used due to its characteristics of the low noise, low input bias current, high CMRR, and low power, this make it well suited for medical applications, such as ECG and noninvasive blood pressure monitors.

Implemented circuit shown in Figure 1. In it you can see the connections of the right arm, left arm and left leg in three of the nine op amp's placed following Einthoven's relationship, as well as also the precordial and corresponding arrangement for increased referrals.

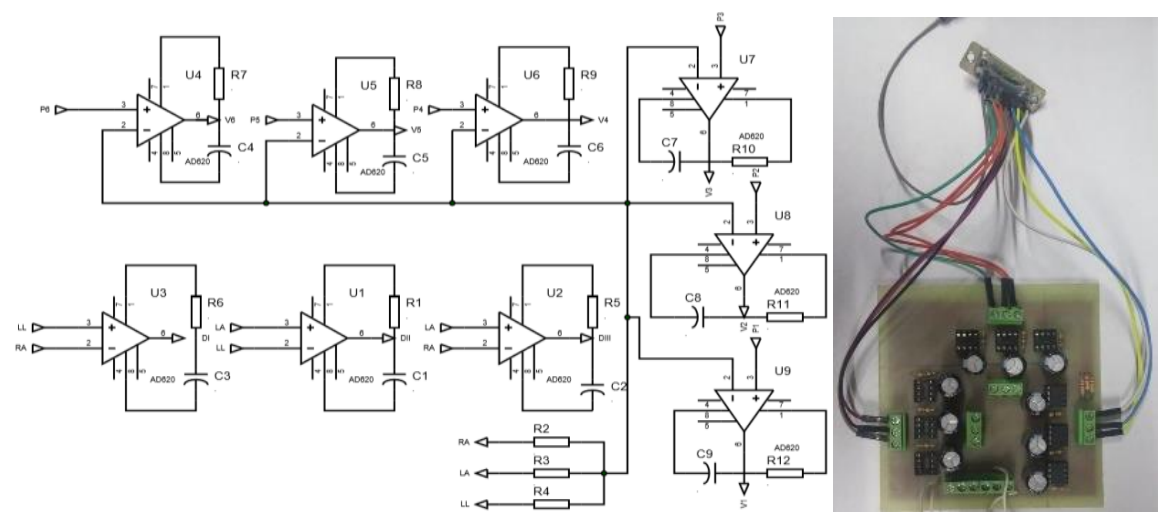

Fig. 1. Schematic and real circuit PCB of the 12-lead electrocardiograph.

The signal is acquired through a series of suction electrodes placed following the AHA system, these electrodes are chosen based on the use of op amps with high input impedance characteristics, it is possible to reduce the effect of polarization, thus avoiding a electric flow which modifies the potential of half cell electrodes, causing distortions in the recorded signal [8]. These signals are transmitted from the patient to the circuit by means of a patient cable to ECG, presented in Figure 2. It is worth mentioning that all power is carried out by a series of connected batteries which gave us the voltage and the electric flow needed to feed the op amps, making the portable system free of any electrical hazard for the patient [9]. 


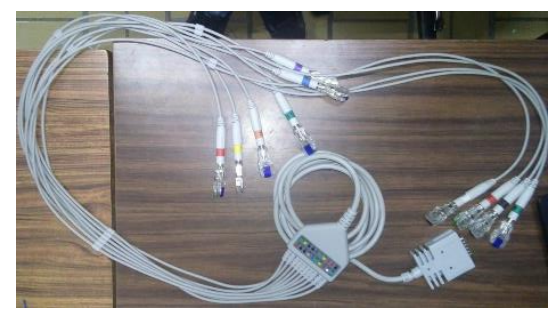

Fig. 2. Patient cable to ECG register.

On the other hand, this test was performed at rest, which was considered to belong to the same frequency range between 0.5 to $150 \mathrm{~Hz}$, so each of the eight lines were subjected to $120 \mathrm{~Hz}$ analog filtering through an $\mathrm{RC}$ array in the gain resistance of the instrumentation amplifier.

This arrangement modified the gain equation present in the datasheet of the AD620. Causing that the amplifier also acted as a band pass filter, when the frequency goes to zero the gain will be 1 , and when it goes to infinity the gain will be approximately 2 . Both gains are despicable when compared with the design gain, this equation is shown in (1):

$$
G=1+\frac{49.4 k \Omega C s}{R_{G} C s+1} .
$$

Eight circuits like this were implemented, to acquire the monopolar branches V1 to V6 and bipolar DI and DII. Then the signals are digitized by means of the USB DAQ6009 card using its eight analog channels. To facilitate the monitoring part of the neural network, high frequency noise was eliminated by configuring the inputs with a sampling frequency of $250 \mathrm{~Hz}$ as shown in Figure 3.

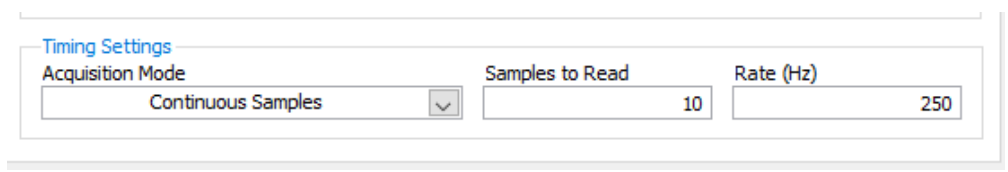

Fig. 3. DAQ-6009 configuration.

Finally, the signals are subjected to a digital filtering process using infinite response to the impulse filters (IIR) to the fourth order impulse, which are: high-pass at $0.5 \mathrm{~Hz}$, low-pass at $120 \mathrm{~Hz}$ and a reject band at $60 \mathrm{~Hz}$. To obtain the 12 derivations from 8 of them, we proceeded to calculate the rest. The derivation DIII was obtained following the relationship between the bipolar derivations, applying the law of Kirchoff voltages obtaining equation (2):

$$
D I I I=D I I-D I .
$$

And for the increased derivations the analysis of the connections for each one of the derivations was carried out, arriving at equations (3-5):

$$
a V R=\frac{-(D I+D I I)}{2},
$$




$$
\begin{aligned}
& a V L=\frac{(D I-D I I)}{2}, \\
& a V F=\frac{(D I I+D I I)}{2} .
\end{aligned}
$$

\subsection{Dynamic Neural Network Design}

Unfortunately, when carrying out the identification, it was difficult to know if it had the external measurements that can be obtained from the system, components due to external or parametric disturbances were presented, this makes the identification process difficult, therefore, the use of the DNN helped us overcome these difficulties. Below is a description of the process of creating the tracking system based on a DNN.

In the tracking of a system, it is assumed that both the input and output values can be measured, so they are a vital part of network training, following the ideas proposed in [10], the mathematical description of the network is given by a differential equation (6):

$$
\dot{\hat{x_{t}}}=A \widehat{x}_{t}+W_{1, t} \sigma\left(\widehat{x}_{t}\right)+W_{2, t} \phi\left(\widehat{x}_{t}\right) u_{t} .
$$

where $\widehat{x_{t}} \in \Re^{\mathrm{n}}$ is the state of the neural network, $u_{t} \in \mathfrak{R}^{\mathrm{q}}$ the input from the model, $W_{l, t} \in \mathfrak{R}^{\mathrm{nxk}}$ the weight matrix of the feedback state layer, $W_{2, t} \in \Re^{\mathrm{nx}}$ the weight matrix of the input layer, $A \in \Re^{\mathrm{n} \times \mathrm{n}}$ a stable matrix selected by trial and error tests, the activation functions $\sigma\left(\widehat{x}_{t}\right)$ and $\phi\left(\widehat{x}_{t}\right)$ are composed of sigmoid functions in each of these equations (7-8):

$$
\begin{aligned}
& \sigma\left(\widehat{x}_{t}\right)=a_{1}\left(1+e^{-a_{2} \widehat{x}_{t}}\right)^{-1}-a_{3}, \\
& \phi\left(\widehat{x}_{t}\right)=b_{1}\left(1+e^{-b_{2} \widehat{x}_{t}}\right)^{-1}-b_{3} .
\end{aligned}
$$

Defining $\Delta t$ as the error between the states of the original system and the states generated by the DNN considered. The DNN weights are adjusted by a set of differential matrix equations, which are equations (9-10):

$$
\begin{gathered}
\frac{d W_{l, t}}{d t}=-K_{l} P \Delta_{t} \sigma^{\mathrm{T}}\left(\widehat{x}_{t}\right), \\
\frac{d W_{2, t}}{d t}=-K_{2} P \Delta_{t} \phi^{\mathrm{T}}\left(\widehat{x}_{t}\right) u_{t}^{\mathrm{T}} .
\end{gathered}
$$

Where $K_{1}$ and $K_{2}$ are constants by means of which the adjustment of the weights is carried out and must be selected by the trial and error method. $P$ is a positive definite matrix that provides the solution of an algebraic Riccati equation (11) described by:

$$
A^{\mathrm{T}} P+P A+P R P+Q=0 .
$$

where the following facts are required to be met:

- There is a positive definite matrix $Q_{0}$ such that the Riccati equation which has a positive solution $P=P^{\mathrm{T}}>0$ 
- It is also required that the $R$ and $Q$ matrices are described by equations (1213):

$$
\begin{gathered}
R=\Lambda_{f}^{-1}+W_{l, t}^{*} \Lambda_{\sigma}^{-1}\left(W_{1, t}^{*}\right)^{T}+W_{2, t}^{*} \Lambda_{\phi}^{-1}\left(W_{2, t}^{*}\right)^{T}+K_{l} \Lambda_{l}^{-1} K_{l}^{\mathrm{T}}+K_{2} \Lambda_{2}^{-1} K_{2}^{\mathrm{T}}+\Lambda_{\xi l}^{-1}, \\
Q=D_{\sigma}+v_{0} D_{\phi}+\Lambda_{l}+Q_{0} .
\end{gathered}
$$

\subsection{Tracking of the Network}

For the operation of the neural network, the algorithm described in equations 6 to 10 was introduced within a control loop by means of the LabVIEW module called "Control Design and Simulation System". As part of the design of the virtual instrument, a selector was use within the algorithm, allowing the user to choose one of the 12 derivations obtained, which enters in the DNN algorithm, thus visualizing the chosen derivation, the tracking of the network, and the error of the same.

\section{Results}

The evaluation of the monitoring of the network can be carried out by placing the prototype of electrocardiograph on the patient, the inclusion of the acquisition card to the computer next to the above-mentioned electrocardiograph and the deployment of the virtual instrument. The patient can view one of the twelve calculated derivations and you will see the corresponding monitoring network as well as the dynamics of the error over the training of the same time.

For example, the DI and DIII connections were followed in an apparently healthy 22-year-old patient, observing the results through the front panel of the program developed in LabVIEW, as shown in Figure 3.

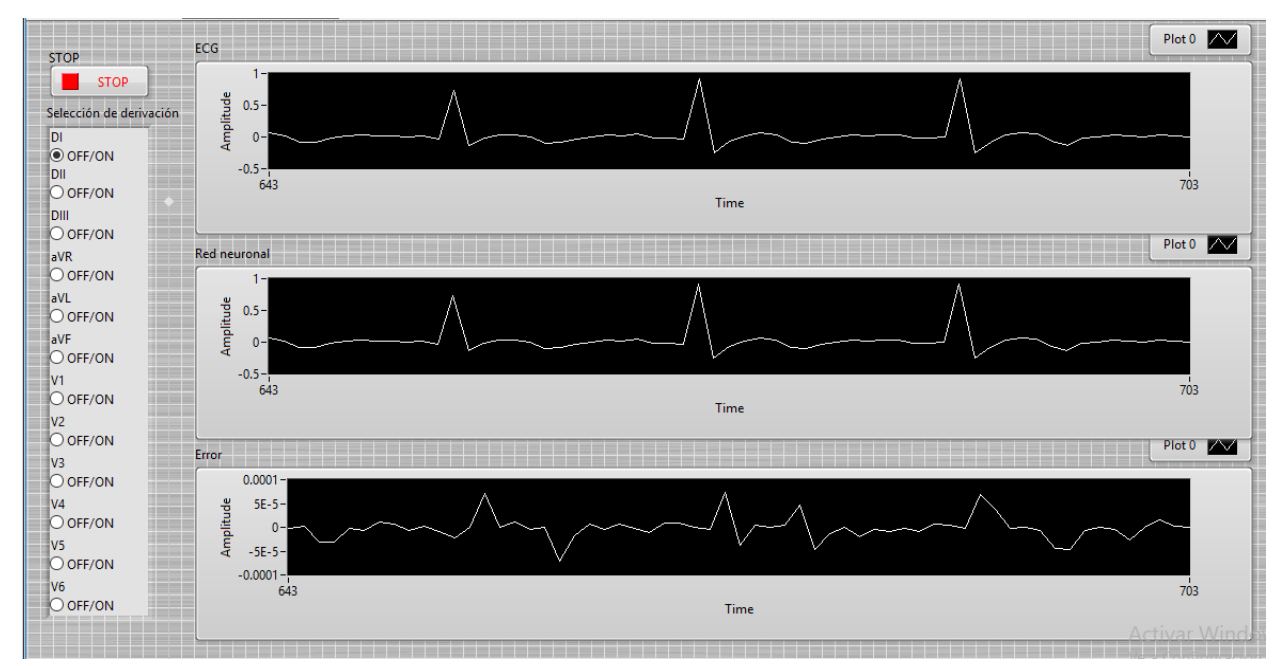

Fig. 3. Front panel of the program in operation. 
The signal and the monitoring of the network are shown in the Graphs 1 and 2, where the ECG signal is represented by the dotted line and the network behavior by the hyphen line. As we can see, a total splicing is observed between both, taking as an example two selected derivation.
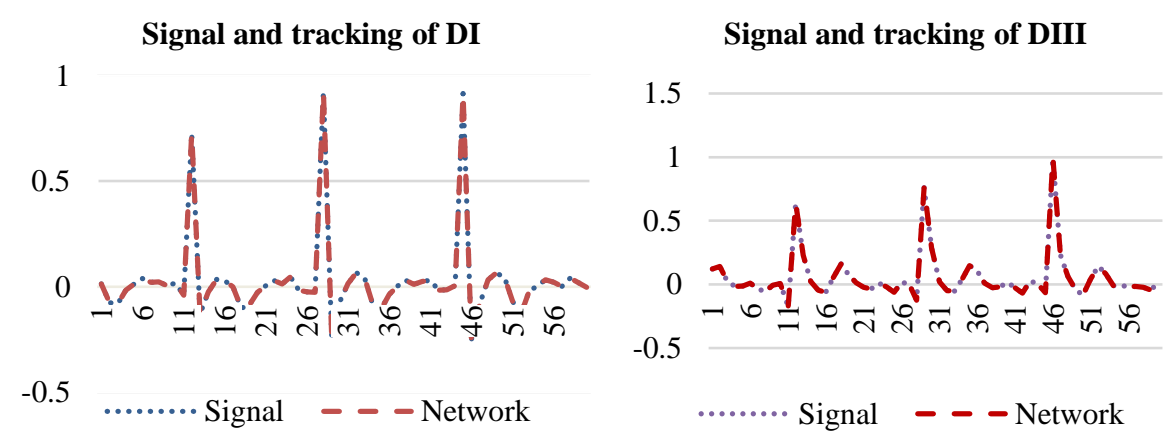

Graphs 1, 2. The signal (dotted line) and the monitoring of the network (hyphen line) in DI and DII.

As we can see in the Graphs 1 and 2, it is impossible to detect the difference between the real values of the signal and the values generated by the network. In both you can see how the network manages to reproduce very well the smooth and abrupt variations in the ECG signal.

Now in the Graphs 3 and 4, the calculation of the error between the values of the ECG signal of the values generated by the DNN is shown. This indicates a good performance of the network because it can reproduce the signal of the derivations shown with errors of approximately \pm 0.0001 . From this we can say that the use of DNN is an excellent alternative for tracking of electrocardiographic signal.

Tracking error of DI

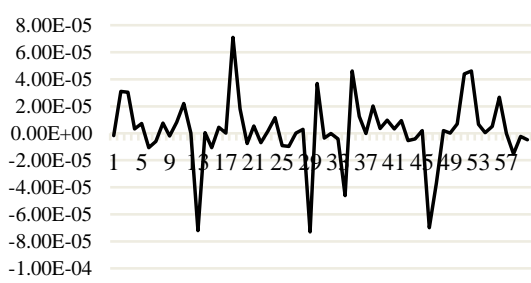

Graph 3. Error of the network in the tracking of DI.
Tracking error of DIII

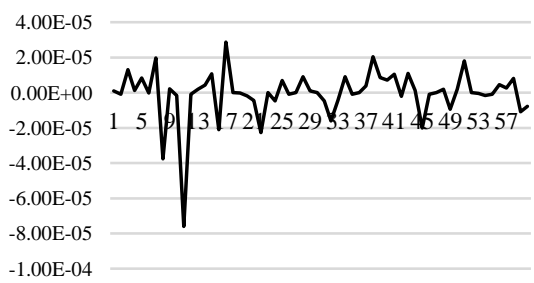

Graph 4. Error of the network in the tracking of DIII.

\section{Conclusions}

In the present paper, a tracking system that uses differential neural networks on line applied to biological signal particularly the ECG obtained from a healthy patient has shown a good performance. In these signals the error obtained by the difference 
between the DNN generate signal and the ECG measure signal is approached asymptotically to zero. The tracking process is shown, and the error signal value less to $10^{-4}$ units was obtained. The methodology based on the DNN, showed that even with abrupt changes, such as those experienced between each peak of the signal, it was possible to obtain almost immediately and precisely such variations, although these were of very short duration. It is considered as future work to expand the functionality of the network to convert it into a prediction system, considering the corresponding changes in the network.

\section{References}

1. Poznyak, A.S., Sánchez, E.N., Yu, W.: Differential Neural Networks for Robust Nonlinear Control. World Scientific, pp. 215-251 (2000)

2. Cabrera, A.I., Poznyak, A.S., Poznyak, T., Aranda, J.S.: Identification of a Fedbatch Fermentation Process: Computational and Laboratory Experiments. In: Bioprocess and Biosystems Engineering. Springer. vol. 24, No. 5, New York: Clarendon, pp. 319-327 (2002)

3. Cabrera, A.I., Aranda, J.S.: Estimating the Trehalose Cytoplasmatic Content during a baker's yeast. In: 10th International Symposyum on Computer Application on Biotechnology. Cancún, México, IFAC (2007)

4. Aguilar, N.C., Chairez, I.: Neuro Tracking Control for Immunotherapy Cancer Treatment. In: IJCNN '06 International Joint Conference on Neural Networks. IEEE, Vancouver, BC, 5316-5323 (2006)

5. Kligfield, P., Gettes, L.S., Bailey, J.J., Childers, R., Deal, B.J., Hancock, E.W., Mirvis, D.M.: Recommendations for the Standardization and Interpretation of the Electrocardiogram. Journal of the American College of Cardiology, pp. 1109-1127 (2007)

6. Ponce, P.: Inteligencia Artificial con Aplicaciones a la Ingeniería. Alfaomega, pp. 238-242 (2010)

7. National Instruments. Manuals. Retrieved from National Instruments: www.ni.com/pdf/manuals/371303n.pdf (2005)

8. García, M.T., Jiménez, A., Ortiz, M.R., Peña, M.A.: Potenciales bioeléctricos: origen y registro. Universidad Autónoma Metropolitana, Unidad Iztapalapa, pp. 65-79 (1997)

9. Webster, J.G., Neuman, M.R., Olson, W.H.: Medical Instrumentation: Application and Design. $4^{\text {th }}$ Edition. John Wiley \& Sons, Inc. pp. 638-674 (2010)

10. Cabrera, A.I., Ramírez, M.G., Galvez, G.: Independent Neuro-Fuzzy Control System. Elsevier, pp. 237-242 (2005) 